FZMw Jg. 6 (2003) S. 18-45

\title{
The Shapes of Indeterminacy: John Cage's Variations I and Variations II $^{1}$
}

\author{
by David P. Miller
}

$-1-$

\section{Introduction: Indeterminacy and the Nature of the Inquiry}

I am interested in the question: what is determined by indeterminate music compositions? The question may seem inherently absurd, since it is in the nature of such compositions to specify less and less, the greater the indeterminacy they exhibit. The case of John Cage's Variations I and Variations II may put the case most clearly. James Pritchett states that these compositions, the first two in a series of eight Variations, are "the two works that extend this principle [of indeterminacy] to its most refined, pure state". ${ }^{2}$ He provides a succinct distinction between indeterminacy and chance: chance "refers to the use of some sort of random procedure in the act of composition," where indeterminacy "refers to the ability of a piece to be performed in substantially different ways". ${ }^{3}$ Thus, while all of Cage's works since the 1951 Music of Changes were composed using chance operations, only some are also what Cage referred to as "compositions indeterminate of performance," where, in most instances, the realizations may be so varied as to be impossible to recognize as originating from the same score. This is the case with the first two Variations.

$$
-2-
$$

About indeterminate notations, Judy Lochhead writes, "The composer 'undetermines' the traditional process by which a performer reads a notation and produces sound successions determined by a composer. Instead the composer 'determines' a set of rules by which a performer may produce notational symbols which regulate sound production". 4 This

$1 \quad$ Acknowledgements: I wish to extend my thanks to Joan Gale, whose support during a writing retreat enabled me to move this paper beyond scattered, handwritten notes. I am also grateful to those who have generously provided copies of out-of-print or live recordings of Variations I and II: André Chaudron, Caleb Deupree, Cornelius Dufallo, Louis Goldstein and John Prokop.

James Pritchett, The Music of John Cage, New York: Cambridge University Press 1993, p. 119. Pritchett, The Music of John Cage, p. 108.

Judy Lochhead, "Performance Practice in the Indeterminate Works of John Cage.", Performance Practice Review 7/1994 (2), p. 234. 
suggests a notion of indeterminate compositions as consisting of rule sets, analogous to the metaphor of "toolkit" often applied to Cage's works in this form. But no matter how generalized it may be, a set of rules, like a toolkit, nevertheless has its specific content. As a score, it will consist of a particular (set of) notation(s) and no other(s), whether they are written, graphical, or (as in the Variations) a combination of the two. Even the most indeterminate work, if distinguishable as a named work at all, does not simply point one in the direction of the entire universe of possibility. Any specific set of instructions and symbols must necessarily constrain one's subsequent choices, even if this constraint happens at a very high level of generality.

$$
-3-
$$

Variations I and Variations II are very similar, yet not identical, as scores. That is, they are distinguishable even if the differences appear, on first encounter, to be slight. What are the differences between them? What do those differences determine, or allow? And, since the works have so much in common, what is determined by their common elements?

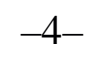

I wish to attempt an analysis of Variations I and Variations II from the perspective of unearthing the constraints their specific notations require, as well as the freedoms they allow. This analysis will, to an extent, draw on the important writings of James Pritchett and Thomas DeLio. Additionally, I will provide a description of the processes I used in making realizations of these works of Cage between 1996 and 1998: two versions of Variations I for two voices (in collaboration with Larry Johnson), and a version of Variations II for sounded mundane objects. All these realizations were performed at Mobius, an interdisciplinary artists' space in Boston, Massachusetts. The description of these specific realizations is intended to provide a concrete counterpoint to the more abstract analysis of the scores.

$$
-5-
$$

\section{The Variations Scores}

The score for Variations I consists of one page of written instructions and six transparency squares. One of the transparencies has twenty-seven points, of four different sizes, marked on it. The other five transparencies each have five intersecting lines drawn on them. The points represent sounds, or sound events, of differing degrees of complexity: the smallest points represent single sounds, while the largest points represent events consisting of four 
or more sounds. The lines represent sound parameters, described by Cage as "lowest frequency, simplest overtone structure, greatest amplitude, least duration, and earliest occurence [sic] within a decided upon time". ${ }^{5}$ It is up to the performer to decide which line represents which parameter, by deliberate choice or some other means. The parameters of each sound in a sound event are determined by placing the transparency with points in some relationship to one of those with lines, and making a perpendicular measurement from the point to each of the lines. Thus, the closer a point is to a line, the lower will be the sound's frequency, the simpler its overtone structure, the greater its amplitude, etc. If a point lies directly on a line, the sound determined would exhibit the maximum possible value for that parameter under the circumstances. Cage does not specify how the transparencies are to be placed, nor does he specify the medium ("Any number of performers; any kind and number of instruments").

$$
-6-
$$

Variations II consists of six transparencies with a single line segment each, and five transparencies with a single point each. (Actually, the transparencies need to be cut apart after the score is received from the publisher.) The points are all the same size. The lines represent "1) frequency, 2) amplitude, 3) timbre, 4) duration, 5) point of occurrence in an established period of time, [and] 6) structure of event (number of sounds making up an aggregate or constellation)". ${ }^{6}$ (See Figure 1.) The function represented by points of different sizes in Variations $I$ - the complexity of a given sound event - has here been taken over by the addition of a sixth line. Cage states that "the sheets are to be superimposed partially or wholly separated on a suitable surface." This may require extending the lines, in order to make a perpendicular measurement, where the transparencies are widely separated. A set of thirty measurements (not the same as thirty sound events) can be made from a single reading of all transparencies used together. If more measurements are necessary, one is to "change the position of the sheets with respect to one another before making them." Measurements can also be made to answer any other questions which arise "regarding other matters or details." Again, the medium of performance is not specified ("for any number of players and any sound producing means").

$5 \quad$ John Cage, Variations I, New York: C.F. Peters 1958.

$6 \quad$ John Cage, Variations II, New York: C.F. Peters 1961. 
Fig. 1

Variations II: one possible configuration of points and lines.

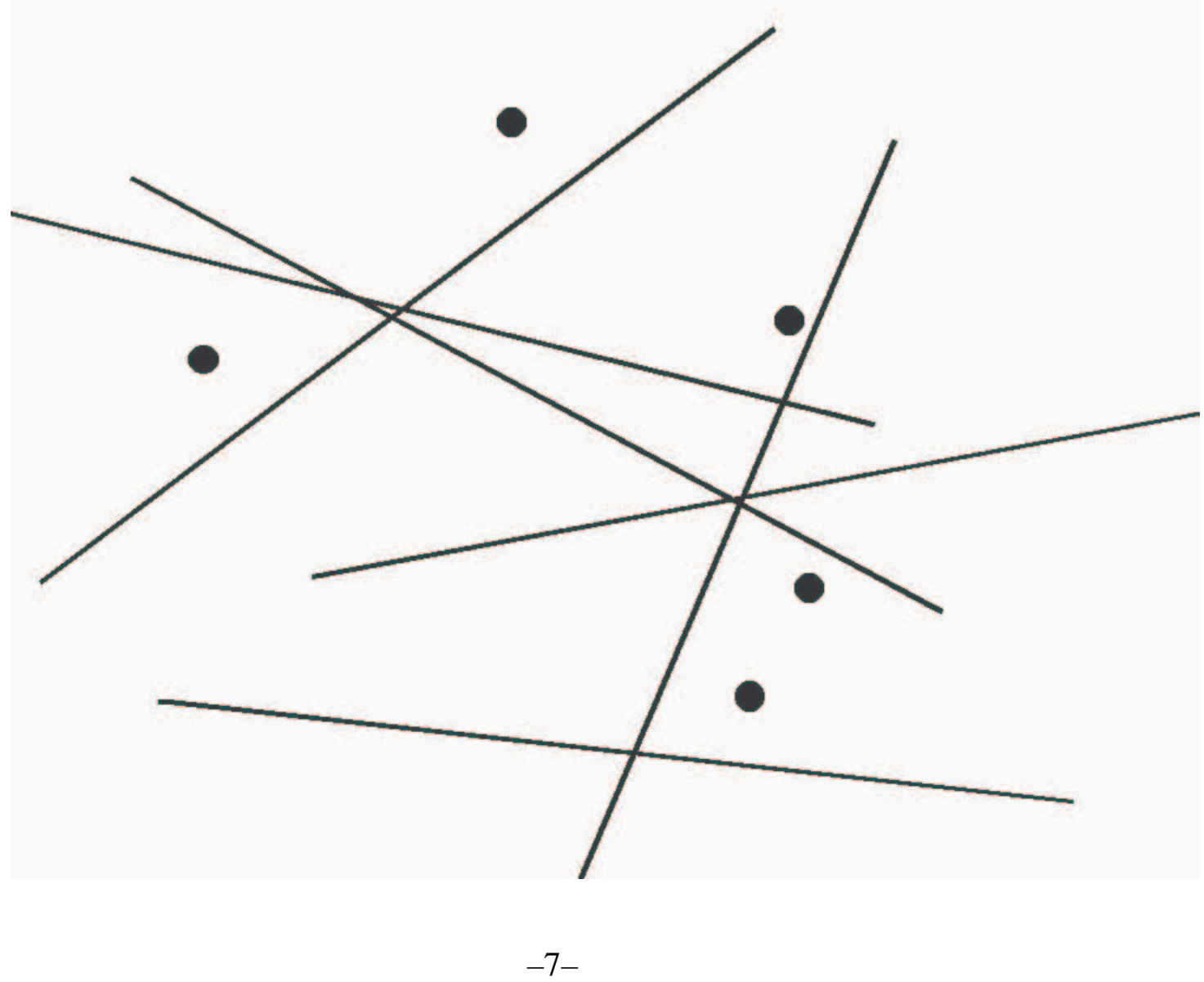

The two Variations scores thus both feature the act of measuring perpendicular lines as the basic means of determining a multitude of sound event parameters. Graphically, both use transparencies on which are drawn points or lines, and which are to be overlaid or juxtaposed in space. Five of six parameters are specified by lines in both of the scores, with the sixth (complexity of the event) indicated by point size in one case (Variations $I$ ), and an additional line in the other (Variations II). Neither score specifies the number of performers, the medium(s), nor the overall length of the performance. The differences between the two scores, and their impacts on realization of the works, will be discussed in greater detail below. 


\section{Two Realizations of Variations I}

\section{A. Common elements}

The first question that one faces in working with either of these scores is the means used to place the transparencies. I acted as spontaneously as possible - that is, with as little thought as possible - both when placing transparencies relative to each other and in assigning parameter values to the lines. Although chance operations were not involved at this stage, the consequences of such spontaneous choices are far too complex to foresee, so that the factor of personal taste is trivial and essentially nullified. These choices were made quickly and simply, without allowing myself to anticipate any potential inconveniences or difficulties.

$$
-9-
$$

Another fundamental decision involves the means of measurement between points and lines, and in fact this decision had continuing ramifications which I did not at first anticipate. Although Cage states that the distances may be "simply observed" (or "eyeballed"), I chose to make detailed measurements using a millimeter ruler, and for the first realization, derived complex numerator/denominator values, as will be seen by the examples discussed below. I chose to start with such fine-grained measurements, not because I assumed I would be able to realize minute distinctions in duration etc. in performance, but because I felt that approximation at any stage reduces the possibilities for precision later on. It seemed to me that using "simple observation" would result in fairly gross values for all parameters at the start (as in "hmmm, that looks like about 40\%"), flattening the landscape of possibility.

$$
-10-
$$

As noted, both versions of Variations $I$ were realized for two voices and performed by Larry Johnson and I. Larry Johnson compiled a set of 19 vowel and 31 sustainable consonant sounds, including phonetic sounds not used in English. The sounds were notated using the International Phonetic Alphabet. Using SoundHack software, Landon Rose produced sonograms of each sound, as pronounced separately by Larry and I. We each used color printouts of our own sonograms as visual surrogates, to order the sounds for complexity. Use of such surrogates again involved some degree of subjective choice, as it 
was frequently difficult to determine which of two sounds was more "visually complex" (although patterns near the ends of the spectra were clearly distinct). But similar to the subjectivity involved in arranging the transparencies, the impact of these choices once applied to the measurements was unforeseeable, and there was no compensation - no rearrangements in the continuum of timbre - made for later inconvenience or difficulty.

$$
-11-
$$

After choosing the human voice as the medium for Variations I, we soon discovered that the factors of timbre, pitch, and amplitude were interdependent. As examples, each of us could only produce a particular timbre within a given pitch range; different timbres also allowed different maximum amplitudes. These parameters could not be "programmed" independently. By contrast, since there seemed to be no good reason to limit any given sound event to a single breath, duration became an independent variable.

$$
-12-
$$

The interdependence of timbre, pitch, and amplitude meant that the concept of an "anchor" parameter (or point of reference) came into play at different stages in the process of preparing the score. For the most part, I chose timbre as the anchor. I determined pitch and amplitude ranges separately for each timbre, and applied the measurements for pitch and amplitude after first determining which timbre would be used for any given sound. However, in order to produce sets of sonograms that we felt confident comparing on the basis of timbre alone, we let pitch serve as the "anchor." Each of us used the same pitch for recording each of fifty sounds, and attempted neutrality in amplitude. (Amplitude alone was observed when recording unpitched sounds.)

$$
-13-
$$

The parameters of duration and beginning point of an event within a period of time (or "earliest occurrence") are also interdependent, at least to the extent that the greatest duration cannot be greater than the time available from the earliest occurrence to the end of the performance. We decided that the concept of "period of time" be considered as the entire chosen length of the performance - twenty minutes - rather than units of time within that total length. The beginning points of events would then be placed on a scale of relative starting times, e.g. starting when $19.7 \%, 65.6 \%$, etc., of the total time had elapsed. (It would be theoretically possible for a performance of one sound event to have a duration of $100 \%$ of the time available, or twenty minutes in this case.) These beginning points would 
then mark off the actual, asymmetrical units of time into which the total performance length was divided, with starting times of e.g. 3'57", 13'7", etc. The maximum duration of each event (equivalent to a measurement of 100\%) was also determined by the differences between subsequent beginning points. In the case of clusters, or multiple-sound events, the beginning point of each sound was mapped to the subunit of time available for the cluster, rather than the total length of the performance. This also determined the order of sounds within a cluster.

$$
-14-
$$

\section{B. Differing Procedures}

As mentioned, I used a millimeter ruler to make all measurements for Variations $I$. For the first realization, these measurements took the form of fractions. The denominator of each fraction was the distance in millimeters from the given point, through the line, and beyond to the furthest edge of the overlaid or juxtaposed transparencies (the sheet of points and the chosen sheet of lines). The numerator of each fraction was the distance from the line to the point. (See Figure 2.)

Fig. 2:

Variations I: fractional measurement technique. Values obtained: $\mathrm{a} / \mathrm{a}+\mathrm{b}$ and $\mathrm{x} / \mathrm{x}+\mathrm{y}$.

[CORRIGENDA, FZMw ed.]

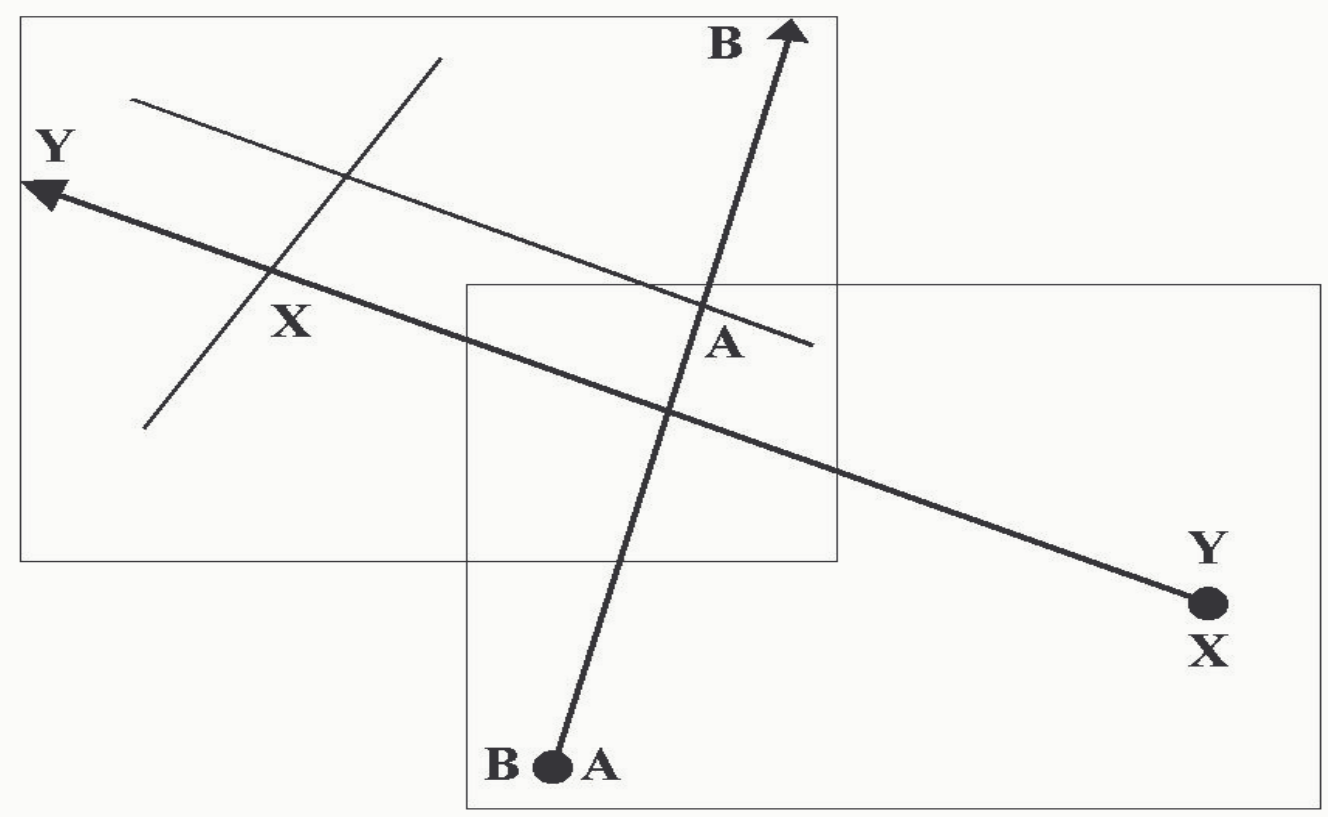


For the sake of intelligibility, the fractions were translated into percentages. A frequency measurement of $77 / 155$, for example, was equal to .49677 , or very slightly below the midpoint of the frequency range determined for a given timbre. An occurrence measurement of 106/163 was equal to .6503 , meaning that a sound event would begin at $65 \%$ of either the total performance time (in the case of single-sound events) or within a subunit of time (for an event within a cluster). Actual examples are given in figure 4 (page 26).

$$
-16-
$$

This approach to determining values had an unexpected result: that a measurement of $100 \%$, equivalent to the value most "opposite" to that assigned by Cage to a line, could not occur. Recall that, in Variations I, lines are not only assigned to parameters, but are also given qualities such as simplest, greatest, least. In this case, if a line was assigned the parameter of (lowest) frequency, a value of highest frequency could not be derived. This is because Cage did not draw any points at the edges of transparencies. This would have been necessary for there to be a 1:1 relationship between the numerator and denominator: 77/77, $157 / 157$, etc., and therefore a value of $100 \%$. If a point fell on a line, which was not uncommon, then the extreme of the quality as specified by Cage (lowest frequency) would be represented. Since Cage does not specify how measurements are to be interpreted, this outcome is one example of an interaction between the specific elements of the score as an artifact, and the means chosen to realize the score. Even at this level, the possibility of indeterminacy is bounded by the concrete nature of the object that Cage produced.

$$
-17-
$$

For the second realization of Variations I, I used the original sets of measurements, but discarded the denominators of each fraction - the distance from a point to the edge of the transparency. Instead, I used the former numerators - the distances from points to lines - to generate a range of absolute values for each parameter. (See Figure 3.) This self-generating set of values would necessarily include greatest as well as least values; that is, for each parameter, there would always be at least one measurement equal to $100 \%$. The same set of measurements, therefore, resulted in different sets of values, and a significantly different realization of the piece. The total duration of 20 minutes was retained, as was the source material, a given set of vocal sounds and the visual ranking of their sonograms. 
Fig. 3:

Variations I: self-generated values technique. Values obtained: a and x.

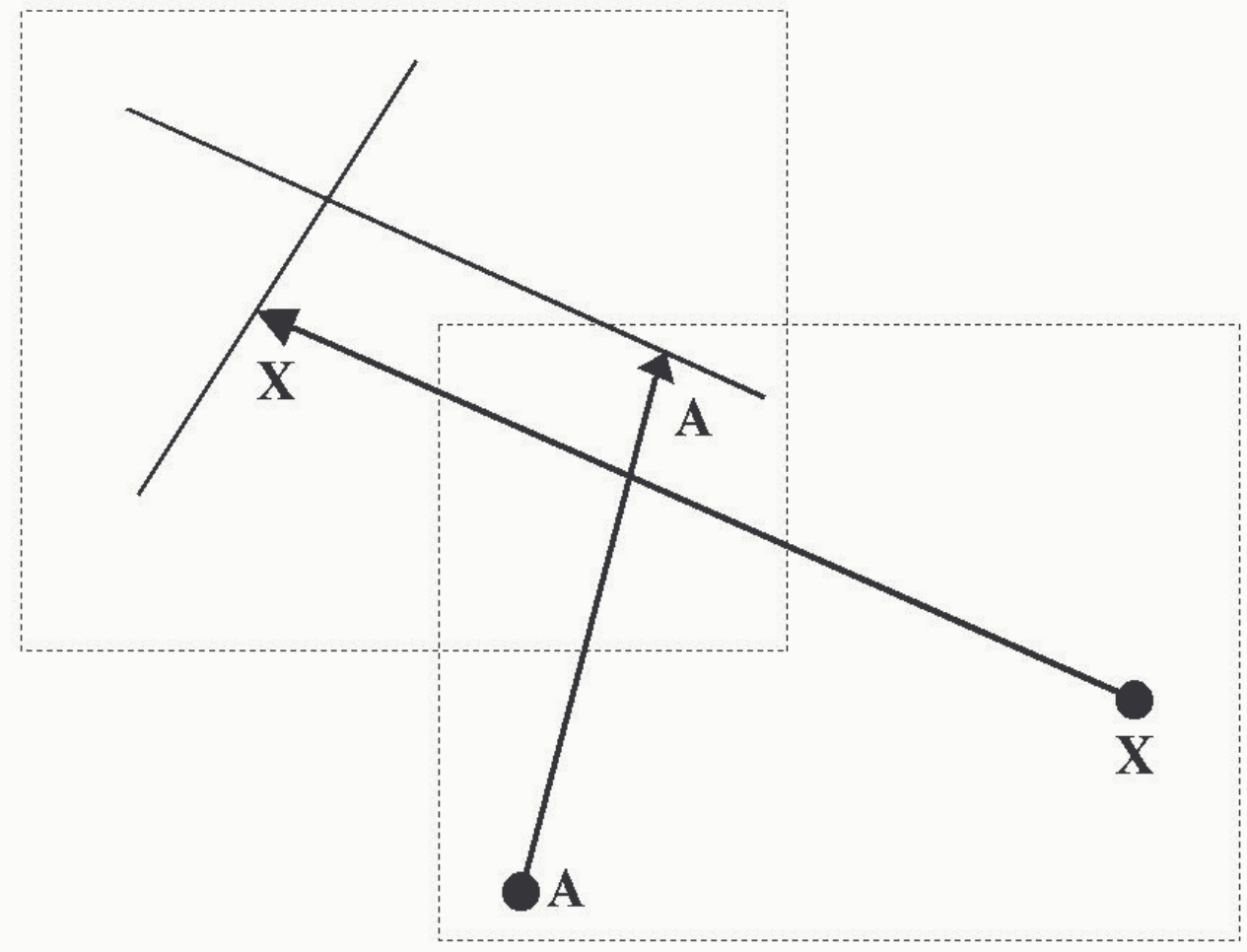

Fig. 4.

Two single-sound events, derived from the same base measurements.

\section{Event A1}

Frequency measurement: 4/55 mm.

Amplitude: 28/145

Duration: $30 / 193$

Overtone structure: $51 / 160$

Occurrence: $27 / 163$

First realization (fractional method):

Overtone/timbre: Scottish "guid" vowel sound, notated as letter u with strikethrough. Frequency: G2. Occurrence: 3'19". Duration: 6". Amplitude: 80\%. 
Second realization (self-generated range of numerator values):

Overtone/timbre: High front rounded vowel sound, notated as lower-case letter y. Frequency: G2. Occurrence: 2'11". Duration: 1". Amplitude: 78\%.

\section{Event A2}

Frequency: $46 / 89$

Amplitude: 78/118

Duration: $131 / 164$

Overtone structure: $35 / 77$

Occurrence: $132 / 160$

First realization:

Overtone/timbre: "M" hum. Frequency: A3. Occurrence: 16'30". Duration: 2'48". Amplitude: $35 \%$.

Second realization: Overtone/timbre: Low front unrounded "A" sound, as in Bostonaccent "park." Frequency: C\#3. Occurrence: 11'26.4". Duration: 18.8". Amplitude: $34 \%$.

$$
-18-
$$

The second realization of Variations $I$ also differed from the first with regard to the construction of clusters, or multi-sound events. The occurrence of sounds within each cluster was mapped, relative to the total amount of time available for the cluster, as in the first version. The duration of each sound within a cluster, however, was determined relative to the time remaining after its occurrence. In the first realization, total time for each cluster was divided into subunits, and durations were mapped to those subunits. The change in procedure in the second version frequently resulted in overlapping sounds within clusters, as the occurrence (or attack) of one sound fell within the duration of another sound (or two). In performance, this led to experiments with combining, blending or oscillating between sounds during the period of overlap. In this way, some clusters could be distinguished in quality from single-sound events, although many cluster sounds were isolated in time nonetheless. 
These changes in procedure in the second realization produced interesting examples of inconvenience and difficulty immediately, the most challenging of which arose at the conclusion. Since each parameter would have at least one measurement each of greatest and of least value, it followed that at least one sound event would necessarily occur at the last possible instant. This would be the instance of latest possible occurrence. It transpired that this event was also an instance of greatest complexity: the point measured from was the largest of the four sizes drawn by Cage, meaning in this case a cluster of four sounds. It was difficult to imagine how I would manage to produce four distinctly articulated sounds in the briefest perceivable instant of time, and in fact I didn't. The solution I adopted was to assign the last second of the performance, rather than the last perceivable instant, to this cluster. While this provided little enough relief, it at least made the task approachable.

$$
-20-
$$

\section{Variations II}

\section{A. Changes between I and II, and their context within Cage's oeuvre}

The points in Variations $I$ are already events, in that their different sizes inherently specify the parameter of complexity. ${ }^{7}$ As a result, when making measurements relative to a given point in Variations $I$, the implied question is "What are the other characteristics of this (one-sound, two-sound, etc.) event?" In Variations II, the situation is quite different. The points have no qualities: the parameter of "complexity" has been removed from the points and given to a sixth line. They are not already defined as events, therefore, but are simply given as points of reference for measurement.

$$
-21-
$$

Similarly, while the lines in Variations I have no predetermined qualities - any of the five parameters (pitch, duration, amplitude, attack, decay) can be assigned to any line proximity to the lines is associated with values such as lowest, simplest, and greatest. The value assigned to each parameter measured for each sound is to be determined according to its position along a continuum: e.g., from simplest to most complex, earliest to latest. In Variations II, since proximities have no values assigned to them, the distance from a point

Pritchett notes this as also true of the notation labeled as BV in the earlier Solo for Piano of the Concert for Piano and Orchestra, 1957-58 (James Pritchett, David Tudor's Realization of John Cage's Variations II, http://www.music.princeton.edu/ jwp/texts/Var2.html, viewed October 31, 2000). 
to line may be given various meanings with regard to the parameter assigned to that line. In other words, the notion of continuum is removed, or made optional, as a metaphor for relating measurements to values. All we know from the score itself is that one line will have to be assigned the parameter of "amplitude," another one "duration," and that for each sound event some means of relating a measurement to a value will have to be determined. As an alternative to the concept of continuum, one may work with a gamut, where a range of values, not necessarily continuous, are assigned positions in an array. (See Figure 5.) Cage's earlier Fontana Mix (1958) suggests this approach. In this work, a graph of 20 x 100 squares is used to determine the "time bracket" of an event using the x-axis, and a variety of other parameters using the y-axis. Using the example of "sound source(s)," Cage suggests that they may be related to the units along the y-axis after being "categorized and related quantitatively to 20." There is no suggestion here of a qualitative arrangement (or continuum of choices), only that the possibilities be assigned positions along the y-axis.

Fig. 5:

Continuum vs. gamut.

\section{Continuum of values}

"Greatest amplitude" (Cage)

Least amplitude (implied)

\section{Gamut of values (example)}

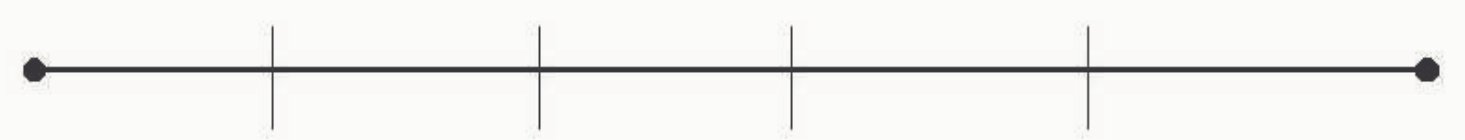

Ampl. 1 Ampl. 7 Ampl. $12 \quad$ Ampl. $9 \quad$ Ampl. 3 
The progress from Variations I to Variations II - removing the parameter of complexity from points, making all points and lines notationally independent, and detaching value from proximity - expresses the greater freedom Cage sought to give the performer, even in comparison with his own earlier works. In 1965, he told Richard Schechner and Michael Kirby that

"while I was at Wesleyan, in the first piece I had had five lines on a single transparent sheet, though I had had no intention of putting them the way I did, I just drew them quickly. At Wesleyan while talking to some students it suddenly occurred to me that there would be much more freedom if I put only a single line or a single notation on a single sheet." 8

\section{$-23-$}

Taking a view further back into the past, James Pritchett notes that the progression of unpacking values from point-and-line notation can be observed beginning with the notations labeled $\mathrm{BB}$ and $\mathrm{BV}$ of the Solo for Piano, from the Concert for Piano and Orchestra (1957-58). ${ }^{9}$ Notation BB presents a fixed set of lines and points in a single image, with parameters assigned to the lines. Notation BV also presents a set of lines and points in a predetermined relationship, but the assignment of parameters has already been removed. (See Figure 6.) At the other end of the spectrum, the transparencies of Variations II imply an altogether open field for determining the characteristics of sound events, "the most flexible tool composition that Cage ever created". ${ }^{10}$

Michael Kirby \& Richard Schechner, "An Interview with John Cage." Tulane Drama Review 10/1965 (2), p. 64.

$9 \quad$ Pritchett, Music of John Cage, p. 135-136.

10 Pritchett, Music of John Cage, p. 136. 
Fig. 6:

Notations BB and BV, Solo for Piano.

(C) C.F. Peters New York $1958^{11}$

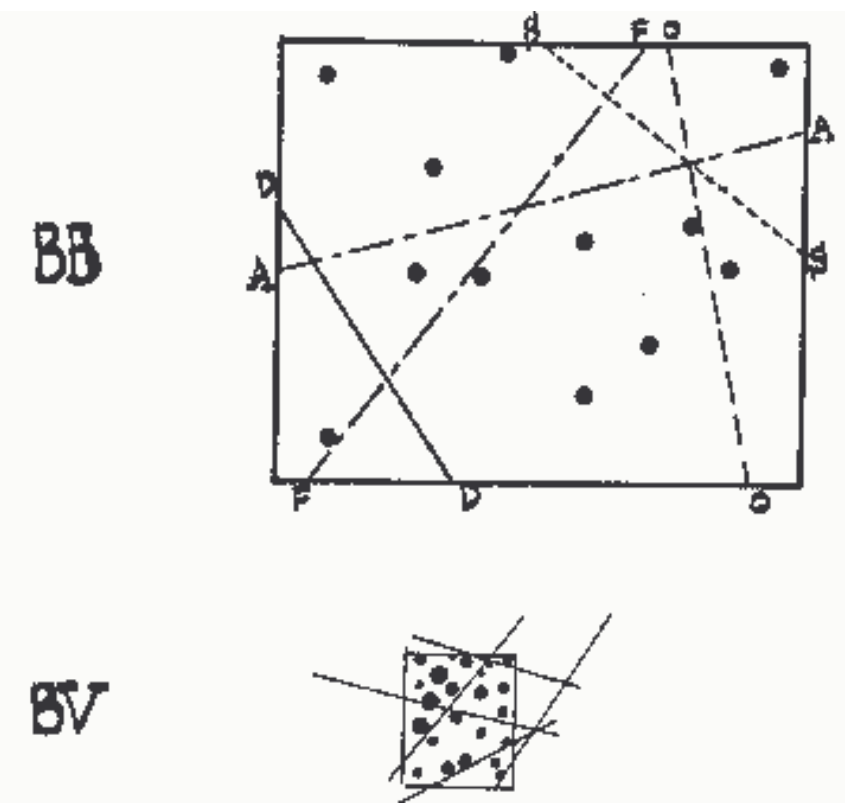

$-24-$

\section{B. David Tudor's realization of Variations II}

In his absorbing analysis of David Tudor's 1961 realization of Variations II for amplified piano, Pritchett observes that Tudor resolved every measurement for each parameter into a binary choice between two values: simple and complex. He writes,

"Tudor's simplification of the measurement system to a binary choice takes the notation of Variations II away from Cage's conception and into a wholly unexpected realm. For Cage, the sonic parameters were analogous to the dials of an imaginary sound synthesizer; Tudor's 'simple/complex' switches represent a different interface between the musician and sound". ${ }^{12}$ 
While this would have been an extraordinary departure for Variations I, we have seen that adjectives as "least", "earliest", etc., which suggest a continuum (or points on a dial, a similar metaphor) are absent from the Variations II score. Tudor's choice can be viewed as a radical simplification of a gamut approach for which Cage's notes at least provide room.

$$
-26-
$$

It should be said that Cage does not seem to have intended to make the notion of a continuum optional. In For the Birds, Cage comments that Tudor's approach to timbre, dividing all possibilities into simple and complex, implied an approach to Variations II "that would never have occurred to me". ${ }^{13}$ He also remarks, however, that "we cannot measure timbre," removing that parameter at least from those that must be placed along a range of continuous values. ${ }^{14}$ Additionally, the progression, from the Solo for Piano forward, of simpler notations steadily emptied of assigned values, is not in contradiction with also opening up the means for making meaning of measurement. From this perspective, Tudor's binary approach may be an extreme one in terms of making meaning, but is not outside the world of the score as given.

$$
-27-
$$

\section{Variations II at Mobius}

At Mobius in 1998, three versions of Variations I, two realizations of Variations II, and a performance of 4'33" were presented on a program collectively titled Variations and Silences. ${ }^{15}$ My realization of Variations II was for unamplified mundane objects: chairs dragged across the floor, paper torn, a rake scraped across plywood, doors slammed, sets of chopsticks rotated in the hand, and more. This choice of heterogeneous source materials raised many questions about how to apply measurements to given parameters, as well as how to articulate the parameters to begin with. For example, can frequency and timbre be

13 John Cage, For the Birds. Boston, Mass.: Marion Boyars 1981, p. 128.

14 Although it is true that current technologies allow for extremely precise measurement of the dimensions of timbre, their reproduction in performance would also have to be accomplished via technological means.

15 The realizations of Variations I included the second version for voices, by Larry Johnson and I, discussed above, and two different versions realized by Steve Norton (woodwinds), Tom Plsek (trombone), and Janet Underhill (bassoon). Landon Rose performed 4'33" using an amplified sounding bow made of re-bar and piano wire. Larry Johnson realized Variations II for solo violin. My realization for objects was performed by Nancy Allison, Donna Palma Coppola, Mary Curtin, Lorinda Garner, Paul Miller, Jane Wiley, and me. 
measured separately when the same object may be sounded differently? Varying sounds, differing in both timbre and frequency range, can be produced from a cardboard tube, depending upon whether it is struck with a wooden stick, rubbed with a brush, or dragged across the floor. Is the basic unit of measurement, then, the object itself, independent of how it is sounded, or the set of varying sounds producible from the object? After some experimentation, I decided to apply measurements to a given object, as sounded in a specific way. Timbre and frequency were often determined by amplitude, which in many cases resolved to the force with which an object was struck or rubbed. Frequencies were independently determinable in only a few instances (e.g., the two speeds of the electric drill or hair dryer).

$$
-28-
$$

The objects were chosen by sounding objects found at my home and at Mobius. I chose objects which interested me considered in isolation, without regard for their possible effects in combination. This is an instance of the exercise of taste at one stage of the process similar to the spontaneous but deliberate placement of transparencies - carried out in the knowledge that any preferences are likely to be confounded to some extent later on. Seventeen objects were chosen:

1. Audience chairs with metal legs (dragged across the wooden floor)

2. A broom (swept across the floor)

3. Three casters on the bottom of an overturned cart (spun)

4. Paper (torn)

5. An oval metal bowl (struck against a table)

6. Long thin pieces of painted wood (rubbed against the edge of a table)

7. A two-speed battery operated drill

8. A two-speed hair dryer

9. A cardboard tube (struck by a wooden dowel)

10. Styrofoam tubes (rubbed together)

11. Bubble wrap (rubbed against itself)

12. A wooden rake (scraped across a piece of plywood set against the wall)

13. A scrub brush (rubbed across an open wooden box)

14. A two-speed large floor fan 
15. Doors (closed or slammed)

16. A large plastic bowl (the rim rubbed against a brick wall)

17. A handful of wooden chopsticks

$$
-29-
$$

I was at first uncertain of how to order these sounds according to timbre, believing that I needed to rank them as I had the speech sounds for Variations I, from least to most complex. I tried to develop a table which would provide a kind of ranking for these sounds considered along the dimensions of timbre, pitch, and volume. But given the radical heterogeneity of the objects, this quickly seemed a forced approach (and in fact I was not at all sure of what information such a table would provide). It was at this point that I realized that the score did not call for parameter values to be arrayed along a continuum. As a result, I developed a gamut approach to measuring timbre, assigning numerical values (1-17) to the sounds, in the order that I had discovered and notated them, as listed above.

$$
-30-
$$

I used the score to determine how many sound events would happen over the course of the 15-minute time length chosen for the performance. Since the notated points do not represent events, one cannot even rely on a strategy of making only as many events for a performance of Variations II as there are points. Once I determined the number of events, I could then make as many measurements as needed, with different arrangements of transparencies. But I did not understand at first how to use the score to determine only one value, that is, a single number. It seemed too much open to deliberate manipulation on my part to set up a combination of transparencies for this purpose alone, so I determined to derive this number from some other operation. The number of events would then be derived as a secondary result, rather than a direct result of an intentional action.

$$
-31-
$$

The process I decided on was to make measurements for five events. With six parameters per event, this provided thirty measurements. The mean average of these measurements (again, the values were measured in millimeters) was taken to provide the number of events in the performance. The number derived was 21 , which easily seemed to be a reasonable number of events for a performance of 15 minutes' length. Subsequent measurements 
determined which of these events were aggregates, and how complex the aggregates were to be.

$$
-32-
$$

I also had to consider the question of the maximum complexity of a sound event. This was related to the number of people available, since I did not wish anyone to sound more than one object at a time: I wanted each person to put her complete attention on each object she was sounding. There were ultimately seven performers involved, so the density measurements were mapped to a range of 1-7, by dividing the range of values obtained into seven equal segments and determining into which segment each measurement fell. The results were:

\section{2 events of one sound \\ 9 events of two sounds \\ 1 event of three sounds \\ 5 events of four sounds \\ 3 events of five sounds \\ 0 events of six sounds \\ 1 event of seven sounds}

$$
-33-
$$

One can observe here that the values are skewed toward the lower end of the range. This obtained as well with the values for timbre, if we take the "lower" end of the gamut as represented by lower-numbered sounds. (The range of values for timbre was divided into seventeen equal segments, and as above, each individual measurement was located in its appropriate segment.) There were, for example, six instances of chairs, eight instances of the broom, and nine instances of castors, but only one instance of doors, three instances of the plastic bowl, and one of the chopsticks. These cases of skewed values could be regarded as anecdotal, but they point to a further, arguably inherent aspect of these scores, to be discussed below. 


$$
-34-
$$

The measurements were applied to each sound in the following order: occurrence, duration, timbre (object), frequency if independently determinable, amplitude. Actions were assigned to the performers not by chance, but according to a couple of criteria. I wished to minimize the handling of multiple objects by the performers, so that each one could become familiar with characteristics of a limited range of objects. I also attempted to distribute the load as evenly as possible (I was not interested in a skewed distribution here), and tried to prevent very brief or hasty transitions between one action and another.

\section{Inherent Characteristics of the Variations}

We are now in a position to more directly address the question raised at the beginning of this paper. What do compositions indeterminate of performance nevertheless determine, by virtue of their inevitably specific characteristics as notations? The first two compositions in the Variations series present three topics for discussion: probable skewed distribution of parameter values, quantitative description of delimited sound events, and the question of intent.

$$
-36-
$$

\section{A. Skewed parameter value distributions}

"Any realization of [Variations II] is the result of a particular configuration fashioned from some superimposition of these sheets. It will be shown that the sonic structure arising from such a superimposition will invariably be that of some statistical correlation of several distributions of sound elements. "16

In Circumscribing the Open Universe, Thomas DeLio provides an analysis of Variations II in a chapter titled "The Morphology of a Global Structure." DeLio uses examples of Variations-like combinations of points and lines, demonstrating that any reading will result in a "statistical distribution of sounds over several parameters and one specific correlation of these distributions". ${ }^{17}$ Any specific reading of this score will manifest in a performance

\footnotetext{
16 Thomas Delio, "The Morphology of a Global Structure: John Cage, Variations II." In Circumscribing the Open Universe. Lanham, Maryland: University Press of America 1984, p. 12.

17 Delio, "The Morphology of a Global Structure: John Cage, Variations II.", p. 15.
} 
where some of the values assigned to sound parameters are more likely to be found in combination than others.

$$
-37-
$$

For the purposes of his discussion, DeLio's illustrations of potential point/line combinations are simplified. He first shows a layout with four points and three lines, the latter representing the parameters of duration, frequency and amplitude only. He also simplifies the measurements into binary choices between short/long duration, low/high frequency, and loud/soft amplitude. His first example, then, illustrates a "realization" with three times as many short/low/loud sounds as long/high/soft ones. In a second example, he demonstrates that a given configuration can be mapped to an $n$-dimensional matrix (where $n$ equals the number of parameters measured). No matter which parameter is assigned to any given axis, the matrix itself is characterized by a fixed structure of correlations. His illustration shows three sound events, with two in a low register and one in a higher register, but with a different assignment of parameters to lines, the events could be characterized by two events of long duration and one of medium duration, etc. DeLio then goes further, showing that dropping six perpendiculars in the physical space used by the score is equivalent to drawing boundaries within the imaginary "space" of all available sound: "the dropping of lines determines the field within which all sonic activity relevant to some particular realization will take place. More specifically, it fixes one specific range of sound from which those to be used in some performance may be chosen". ${ }^{18}$ The fact that his illustrations all use fewer parameters than are provided for in either of the Variations obviously serves the purpose of making it possible to grasp his dense and rigorous arguments. His conclusions can be extrapolated to configurations using the full complement of parameters and more finegrained measurements. (Although, as Tudor's realization demonstrates, and as Cage's allowance of "eyeballed" measurements suggests, fine granularity is not strictly necessary.)

18 Delio, "The Morphology of a Global Structure: John Cage, Variations II.", p. 24. 


$$
-38-
$$

DeLio states that "the score contains within it the full range of all possible configurations of six lines and five dots and, consequently, the full range of statistical structures to which these configurations give rise", ${ }^{19}$ and concludes, "the structure of his composition, then, is not just one specific statistical distribution which he has chosen, but is an entire range of such distributions and the mechanism for choosing from these". ${ }^{20}$ It may be paradoxical, then, to realize that if the act of juxtaposing transparencies is performed without forethought, there are types of outcomes that are unlikely to result, for either of the first two Variations. Among these outcomes are those which show an equal or evenly graded distribution of values: that is, outcomes which do not demonstrate skewed distributions.

$$
-39-
$$

It is possible to manipulate the score for Variations II (and perhaps $I$ ) to produce a performance which, for example, consists of a single, sustained drone, or of a series of major scales, or which has the beat of a Sousa march. These possibilities may reside in the score's global potential, but at the its edges, in a sense. They are outside possibilities, brought about through deliberate intervention. As an example, one might re-place a point, in deliberately calculated increments, relative to the "frequency" line, to create a melody. (See Figure 7.) But if the Variations transparencies are not manipulated toward a specific, desired outcome, any performance will be characterized by an asymmetrical "subspace" derived from the potentially global space of sound available in the performance medium chosen for a realization (voice, violin, torn paper, etc.). This asymmetricality, analyzed by DeLio, was manifested in the Mobius performances, and is evident in every recording or performance of either work in my listening experience. What determines this extremely strong tendency in these indeterminate scores? It is the fact that measurement from points to lines is the basic act involved in realizing the scores. We draw intersecting lines in the imaginary space of available sound, and, as DeLio's title puts it, "circumscrib[e] the open universe." By the actions of preparing the score and measuring it, the performer makes an n-dimensional, abstract subspace manifest in sound, or makes the inaudible audible.

\footnotetext{
19 Delio, "The Morphology of a Global Structure: John Cage, Variations II.", p. 19.
}

20 Delio, "The Morphology of a Global Structure: John Cage, Variations II.", p. 25. 
Fig. 7:

Writing a melody using Variations II.

"Frequency" line

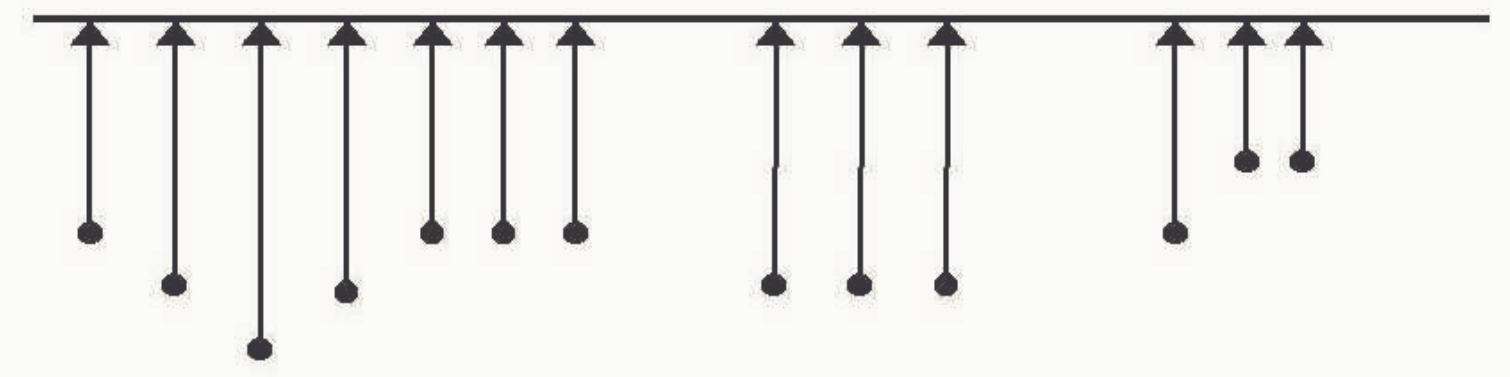

Sequential frequency measurements

$-40$

On the strictly physical plane (of the score as artifact), without deliberate manipulation of the distances between points and lines, it is virtually inevitable that any given point will lie in an eccentric relationship to all of the lines. This is inherent in Variations I, where the eccentric relationships are fixed: there are specific configurations of points and lines drawn on the transparencies, which cannot be regularized. In Variations II, where each set of measurements from a single point to a set of six lines follows a fresh layout of the transparencies, the realization created will nevertheless come from an accumulation of eccentric point-to-line(s) patterns. It is an outside possibility, of course, that any given accumulation of eccentric patterns might result in an even distribution of values, as together the eccentricities cancel each other out. This does not seem to have happened in any of the numerous realizations of these pieces available on recordings.

$-41-$

For my two versions of Variations I, although the change in measurement techniques generated two distinctly different data sets, the phenomenon of a skewed range of values persisted. The second method of measurement (self-generation) produced a set of values where the greatest value $(1 / 1$, or $100 \%)$ for each parameter was determined by an "outlier", with few other measurements approaching this. This meant that, again, the values for each 
parameter were skewed toward smaller percentages, resulting again in a predominance of earlier occurrences, lower frequencies, etc. So, while the same base measurements resulted in two different sets of specific values, and two different scores, the phenomenon of uneven distributions of values remained.

$$
-42-
$$

Figure 8 provides summary data showing the distribution of values for each parameter in my part in the second realization of Variations I. Fifty-two (52) sets of measurements were made. Had the values resulting from measurements been distributed with perfect uniformity, then we would, as a natural consequence, find 26 measurements falling in the lowest one-half of potential values, 39 measurements falling in the lowest three-quarters, 47 measurements in the lowest $90 \%$ and 49 measurements in the lowest $95 \%$. Instead, to varying degrees, the measurements for each parameter are distributed toward the lower end of the range of potential values. For example, the frequency parameter had a maximum value of 202. Had the frequency values been uniformly distributed, one would expect half of the measurements to have values of between 1 and 101. Instead, the lowest $50 \%$ of values fell between 1 and 56, 27.7\% of 202 .

Fig. 8:

Distribution of values in Variations I.

\begin{tabular}{|c|c|c|c|c|}
\hline $\begin{array}{l}\text { Number of } \\
\text { measurements } \\
(100 \%=52)\end{array}$ & $\begin{array}{l}50 \% \\
\text { (26 measurements) }\end{array}$ & $\begin{array}{l}75 \% \\
(39)\end{array}$ & $\begin{array}{l}90 \% \\
(47)\end{array}$ & $\begin{array}{l}95 \% \\
(49)\end{array}$ \\
\hline $\begin{array}{l}\text { Frequency values } \\
\text { (range 1-202) }\end{array}$ & $\begin{array}{l}1-56 \\
27.7 \% \text { of } 202 \\
\end{array}$ & $\begin{array}{l}1-87 \\
43.1 \% \\
\end{array}$ & $\begin{array}{l}1-140 \\
69.3 \% \\
\end{array}$ & $\begin{array}{l}1-156 \\
77.2 \% \\
\end{array}$ \\
\hline $\begin{array}{l}\text { Amplitude values } \\
\text { (range 1-118) }\end{array}$ & $\begin{array}{l}1-38 \\
32.2 \% \text { of } 118 \\
\end{array}$ & $\begin{array}{l}1-63 \\
53.3 \% \\
\end{array}$ & \begin{tabular}{|l|}
$1-93$ \\
$78.8 \%$ \\
\end{tabular} & $\begin{array}{l}1-95 \\
80.5 \%\end{array}$ \\
\hline $\begin{array}{l}\text { Duration values } \\
\text { (range 1-427) }\end{array}$ & $\begin{array}{l}1-81 \\
19 \% \text { of } 427\end{array}$ & $\begin{array}{l}1-131 \\
30.7 \%\end{array}$ & $\begin{array}{l}1-271 \\
63.5 \%\end{array}$ & $\begin{array}{l}1-325 \\
76.1 \%\end{array}$ \\
\hline $\begin{array}{l}\text { Overtone values } \\
\text { (range 1-166) }\end{array}$ & $\begin{array}{l}1-48 \\
28.9 \% \text { of } 165\end{array}$ & $\begin{array}{l}1-75 \\
45.1 \%\end{array}$ & \begin{tabular}{|l|}
$1-97$ \\
$58.4 \%$ \\
\end{tabular} & $\begin{array}{l}1-103 \\
62 \%\end{array}$ \\
\hline $\begin{array}{l}\text { Occurrence values } \\
\text { (range 1-229) }\end{array}$ & $\begin{array}{l}1-86 \\
37.6 \% \text { of } 229\end{array}$ & $\begin{array}{l}1-112 \\
48.9 \%\end{array}$ & $\begin{array}{l}1-163 \\
71.2 \%\end{array}$ & $\begin{array}{l}1-176 \\
76.9 \%\end{array}$ \\
\hline
\end{tabular}


It is important to emphasize that the data presented here, in addition to the previously presented material on skewed distributions in my version of Variations II, can only provide anecdotal evidence. I present it not as definitive, but rather as tending to confirm DeLio's analysis.

\section{$-44-$}

\section{B. Starting point: quantitative measurements and isolated sound events}

Another basic characteristic of these scores is that their starting point is measurability; that is, they do not allow sound to exist without quantitative predeterminations. Even Tudor's simple/complex realization depends upon this quantitative approach. When one enters the world of these compositions, it is easy to take this aspect for granted, forgetting that it is not an inevitable approach to imagining sound. An obvious contrast with this approach would be one process involved in electroacoustic composition, that of taking field recordings and listening closely to discover their innate qualities, with quantitative measurement as a secondary operation, if indeed it is present at all.

$$
-45-
$$

The first two Variations also put a strong emphasis on sounds in isolation, concentrating on a fine level of granularity relative to source materials (such as "phonemes" or "household objects"). The aggregates are themselves built from discrete events, not brought into being all at once, as would happen if several objects were simultaneously dropped on piano strings, or if a group of musicians played a different note at the same moment (cf. the orchestral version of Cage's Ryoanji). Even if a given realization included a passage of melody - a sequence of tones perceivable as a continuity - this could only result from a sequence of isolated events built one by one.

$$
-46-
$$

The text for Variations II does, of course, include the statement that if any other questions arise about a given realization, one may "put the question in such a way that it can be answered by measurement of a dropped perpendicular." The score, then, appears to provide even greater flexibility than it seems at first, leading to James Pritchett's statement that "Variations II is more than a tool, it is a meta-tool." ${ }^{21}$ But is this score really of use when it

$21 \quad$ Pritchett, The Music of John Cage, p. 137. 
comes to answering questions, or providing approaches to composition or performance, which cannot be captured by the act of linear measurement? One is reminded of the saying, "When all you have is a hammer, everything looks like a nail." Consider the following processes:

Cultivate lucid dreaming with regard to sound material.

Conduct a soundwalk through one's neighborhood, focussing on the smallest sounds.

Extract all the elements from an environmental recording which one finds striking.

Transcribe all references to sound in whatever book you happen to be reading.

$-47-$

There seems no use in necessarily subordinating the outcomes of these processes into a frame of point-to-line measurement, even if one may pose a great array of questions to be answered by measurement. Attention to the material resulting from processes such as these will not necessarily lead you in the direction of closely analyzing the technical parameters of individual sound events; other compositions may well result which have nothing to do with this approach.

$$
-48-
$$

\section{The question of intent}

As noted above, Variations II can be more easily directed than Variations I toward a more or less predetermined outcome, due to the complete independence of its graphic elements. One can create a deliberately patterned series of juxtapositions, resulting in melodies, discernible rhythms, and so on, or in a deliberately narrowed range of values (to make, say, a "slow, quiet version" of the piece). An example of manipulation toward creating melody was given above. As another example, a steady drone could be created via a realization which had only one sound event, in which the occurrence of the event began in the first instant, the duration of which was equal to the total time available, using a pitched sound source. Once started on this path, it would indeed be possible to produce, or reproduce, a piano sonata by Mozart. One thousand monkeys with one thousand copies of the score could produce the Symphony of - but enough. 
With regard to a foreseen outcome one must ask: Why bother? How, in spirit or intent, is such an outcome a nontrivial realization of Variations II? The question of intent, or spirit of the work, goes beyond homage to Cage's stated values, though those are hardly to be slighted. Indeed, it seems that using either of the first two Variations in a manner that counters Cage's core value of openness to the unexpected - which one must do to produce a realization not characterized by an asymmetrical sound space - will be characterized by diminishing returns with regard to effort expended. The more painstaking the manipulation involved to aim readings of the score toward a specifically imagined outcome, the more the score is not a tool, or a meta-tool, but an obstacle. Instead of the Variations II score serving as a means to compose any kind of music, it will in such a situation prove to be a superfluous interpolation. If that is one's aim, I would say bluntly: "Do your own work; compose your own music and give it its own name," rather than assigning Cage's name to it.

$$
-50-
$$

\section{Conclusion}

This study began with an interest in discovering the extent and nature of determinate elements in the highly indeterminate Variations $I$ and II. Works of this sort (not to mention Cage's work in general since the Music of Changes of 1951) are sometimes criticized as possessing no inherent qualities, plan, or vision, and therefore not actually qualifying as compositions, or music, at all. In contrast, we have seen that these works possess concrete characteristics which enable the production of music of a particular nature. The indeterminacy of these works remains, of course, and indeed the variety of specific realizations possible is so great that few listeners will recognize them as originating from a common notational source. ${ }^{22}$

$$
-51-
$$

On the artifact level, the actual characteristics of the transparencies (for example, that no points are drawn at an edge) interact with the technique chosen to interpret measurements, to produce different sets of parameter values. In Variations I, some qualities are embedded,

22 At the same time, while a discussion of the existing recorded versions is beyond the scope of this paper, it is possible to state that nearly all of the many with which I am familiar reveal the characteristics analyzed here. 
as it were, in the notation. Asymmetrical distributions and correlations of values are fixed due to the fixed asymmetricality of the notation on each transparency.

$$
-52-
$$

In Variations II, all qualities have been removed from the notation, which may be used to determine a more complex set of parameters for each sound event than is possible in Variations I. In theory, all outcomes are possible, including those showing strict symmetry or patterned distribution of values for any parameter. The greater degree of deliberate manipulation required, however, makes achieving such an end a matter of questionable worth.

$$
-53-
$$

Both works concentrate the performer's and listener's attention on discrete and discontinuous sound events. They also take the action of measuring and the quality of measurability as premises. Finally, when the Variations transparencies are used in a relatively simple and direct manner, with little or no forethought or deliberate manipulation, the result will be a performance characterized by a asymmetrical distribution of parameter values.

$$
-54
$$

It is unlikely that anyone reasonably familiar with Cage's work will mistake a realization of Variations I or II for Litany for the Whale, Europera 5, Solo for Voice 2, Four ${ }^{6}$, Aria, or a great number of other works. The first two Variations are indeed compositions: their many realizations comprise an extended family with a shared inheritance, however divergent their surface appearances may appear to be. 


\section{References}

Cage, John. 1958. Variations I. New York: C.F. Peters. 1958. Concert for Piano and Orchestra. New York: C.F. Peters. 1961. Variations II. New York: C.F. Peters. 1981. For the Birds. Boston, Mass.: Marion Boyars.

DeLio, Thomas. 1984. "The Morphology of a Global Structure: John Cage, Variations II." In Circumscribing the Open Universe. Lanham, Maryland: University Press of America.

Kirby, Michael and Richard Schechner. 1965. "An Interview with John Cage." Tulane Drama Review 10 (2), 50-72.

Lochhead, Judy. 1994. "Performance Practice in the Indeterminate Works of John Cage." Performance Practice Review 7 (2), 233-241.

Pritchett, James. 1993. The Music of John Cage. New York: Cambridge University Press. --------. 2000. "David Tudor's Realization of John Cage's Variations II." http://www.music.princeton.edu/ jwp/texts/Var2.html 